\title{
Raman Characterisation of Single-Walled Carbon Nanotubes Produced by the Catalytic Pyrolysis of Methane
}

\author{
M. Seifi ${ }^{\text {a }}$, D. K. Ross ${ }^{a^{*}}$, A. Giannasi ${ }^{\text {b }}$ \\ ${ }^{a}$ Institute for Materials Research, University of Salford, Salford, M5 4WT, UK \\ b IFAC-CNR, Polo Scientifico Disesto Fiorentino, Via Madonna del Piano, Italy
}

\begin{abstract}
Bundles of Single-Wall Carbon Nanotubes were synthesised using a Chemical Vapour Deposition technique. Methane was used as the carbon-carrier gas. It was decomposed at $950^{\circ} \mathrm{C}$ over a catalyst material consisting of $2 \%$ by wt Fe chemically deposited on an MgO support. For characterising the sample we used Scanning Electron Microscopy (SEM) and Transmission Electron Microscopy (TEM) to show the presence of nanotubes and also to measure their diameters and the size of carbon deposits. Raman Scattering was also used to probe the electronic properties and hence derive diameters of the SWNT. For this reason, Raman spectra of SWNT sample were measured in both the radial breathing mode (RBM) and tangential mode (TM) ranges using three different laser lines. For comparison, similar data has been reported for a standard commercial SWNT material.
\end{abstract}

\section{Introduction}

Carbon nanotubes were discovered in 1991 by Iijima.[1] These tubular structures can be either single-walled (SWNT) or multi-walled (MWNT). In all cases, they consist of seamless cylindrical shells of graphite sheet. The single-walled nanotubes can be either metallic or semi-conducting depending on their helicity and diameter [2]. Carbon nanotubes of all types can be produced by a variety of methods. The most common methods of synthesis are Laser Vaporisation [3] and Electric Arc discharge [4] (both high temperature processes) and Chemical Vapour Deposition (CVD) [5] which is a medium temperature method. We have exclusively employed the CVD method.

\footnotetext{
*Corresponding author. Fax: 0044161 2955147; d.k.ross@salford.ac.uk
} 
This method involves passing a hydrocarbon gas over suitable catalytic particles on an inert substrate. A variety of methods have also been used to purify the materials produced, by dissolving away first the catalyst support and then any exposed catalyst. The purity of the remaining product, i.e. the proportion of the sample consisting of nanotubes, and the range of diameters of the nanotube ropes and of individual nanotubes can be estimated using SEM and TEM.

The distribution of SWNTs radii was characterized using Raman spectroscopy, which is a powerful technique for identifying the properties of individual nanotubes in the material [6]. The radial breathing modes (RBM) of the nanotubes, which appear in the range $100-300 \mathrm{~cm}^{-1}$, and the double peak structure due to the Transverse Modes, which appear in a higher frequency range (1500-1600 $\left.\mathrm{cm}^{-1}\right)$, are characteristic of the spectra from different nanotube geometries [7]. The distributions of diameters and the electronic properties of the nanotubes can therefore be determined from an analysis of these RBM and TM bands.

Magnesium oxide ${ }^{1}$ was used as the catalyst support, the catalyst consisting of nanoparticles of iron. Methane was used as the carbon carrier gas which was decomposed over the metal catalyst at $950{ }^{\circ} \mathrm{C}$.

\section{Experimental Technique}

In developing our production technique, many parameters were varied. Efforts at reproducing good yields indicated which of the experimental parameters were the most critical and how carefully they had to be controlled. The conditions reported here were the best that we encountered but the range of possible parameter choice is so large that they probably only represent a local optimum.

The catalyst materials for our CVD process were prepared by mixing $6 \mathrm{~g}$ of magnesium oxide (MgO) powder, with a surface area of $10 \mathrm{~m}^{2} / \mathrm{g}^{1}$, with $0.87 \mathrm{~g}$ of iron 
nitrate, $\mathrm{Fe}\left(\mathrm{NO}_{3}\right)_{3} \cdot 9 \mathrm{H}_{2} \mathrm{O}$, in methanol solution. The solution was sonicated for about 20 minutes and then the methanol was removed using a rotary evaporator. The catalyst and support were then dried at $140{ }^{\circ} \mathrm{C}$ overnight and ground into a fine powder. The syntheses of the SWNT material were carried out in a horizontal reactor consisting of a quartz tube, $640 \mathrm{~mm}$ long and $50 \mathrm{~mm}$ in diameter. $3 \mathrm{~g}$ of the catalyst + catalyst support were placed in a quartz boat in the centre of the reactor tube. This was then heated in a gas mixture of $\mathrm{Ar} / \mathrm{H}_{2}$ at a flow rate of $100 / 100 \mathrm{ml} / \mathrm{min}$ as the temperature was raised linearly to $500{ }^{0} \mathrm{C}$. By this temperature, the iron nitrate had decomposed into iron oxides $\left(\mathrm{Fe}^{+++}\right.$and $\mathrm{Fe}^{++}$). In order to form elemental iron, nano particles of which can serve as nuclei for nanotube growth, the catalyst was then reduced at a higher temperature in hydrogen flowing at a rate of $100 \mathrm{ml} / \mathrm{min}$ [8]. Then, at a reaction temperature of around $950{ }^{0} \mathrm{C}$, a mixture of $\mathrm{Ar} / \mathrm{CH}_{4} / \mathrm{H}_{2}$ was passed through the system at flow rates of 200/40/100 $\mathrm{ml} / \mathrm{min}$ for 30 minutes. The furnace was then switched off and allowed to cool (unaided) to ambient temperatures. In order to remove the support material and the metal particles, the samples were then treated with acid. First they were placed in dilute $\mathrm{HCl}$ acid for two days. They were then transferred into $\mathrm{HNO}_{3}$ for three days, filtered under vacuum, using a $0.2 \mu \mathrm{m}$ paper filter, and then rinsed with deionised water. After the acid treatment, all the catalyst support and accessible catalyst had been removed. It should be noted that this process was not able to remove particles that had become entirely coated with amorphous carbon. Finally the resultant material was dried at $140{ }^{0} \mathrm{C}$ overnight.

The purified sample was then characterised using an SEM (JOEL JSM 6400), and a TEM (JEOL 3010) and by the Raman Scattering. Different incident wavelength radiations (488, 514.8 and $632.8 \mathrm{~nm}$ ) were used for measuring the Raman spectra. The three significant components of the measured spectra were: (a) Radial Breathing 
Modes (RBM) of the SWNTs around 150-250 $\mathrm{cm}^{-1}$; (b) Tangential Modes (TM) of the tubes between 1500-1600 $\mathrm{cm}^{-1}$ and (c) a peak around $1340 \mathrm{~cm}^{-1}$ due to vibrations of C-C graphite bonds in the carbon nano-particles[9,10]. Each region of the Raman spectra provides information on different properties of the SWNT. The RBM frequencies depend inversely on the tube diameter, $\omega_{\mathrm{RBM}}=\frac{\mathrm{A}}{\mathrm{d}_{\mathrm{t}}}+B .[11]$. The electronic properties of the SWNT can be determined from the TM profile. This profile is generally broad and asymmetric for metallic tubes, in comparison with the sharp and purely lorentzian line shapes observed for semiconducting tubes [12].

\section{Results and discussion}

\section{1. SEM and TEM}

The purified sample was characterised using SEM (JOEL JSM 6400) and TEM (JEOL 3010). With the present catalysts and CVD conditions, we observe a large number of SWNTs bundles in the sample. The SEM images at different points are all very similar to each other. Fig.1 shows the presence of SWNTs bundles with a range of diameters throughout the image, and these images were typical of the whole sample. The diameters of the bundles lie between 10 and $70 \mathrm{~nm}$ (Fig 2). It will also be noted that there are many dark dots. These are due to metal particles encapsulated within the nanotubes and also, within shaded zones, attributed to amorphous carbon [9]. The samples for TEM examination were prepared by sonicating about 3mg of material in alcohol, One drop was then put on the Lacey-Carbon-coated Cu grid. Fig.3 clearly shows the TEM image of SWNTs bundles, where each bundle contains several tubes. The size of the bundles here ranges from 5.3 to $16 \mathrm{~nm}$, and the lengths are up to tens of micrometers. The difference in bundle size between the two images (SEM, TEM) may be attributed to the sonication of the sample prior to examination with the 


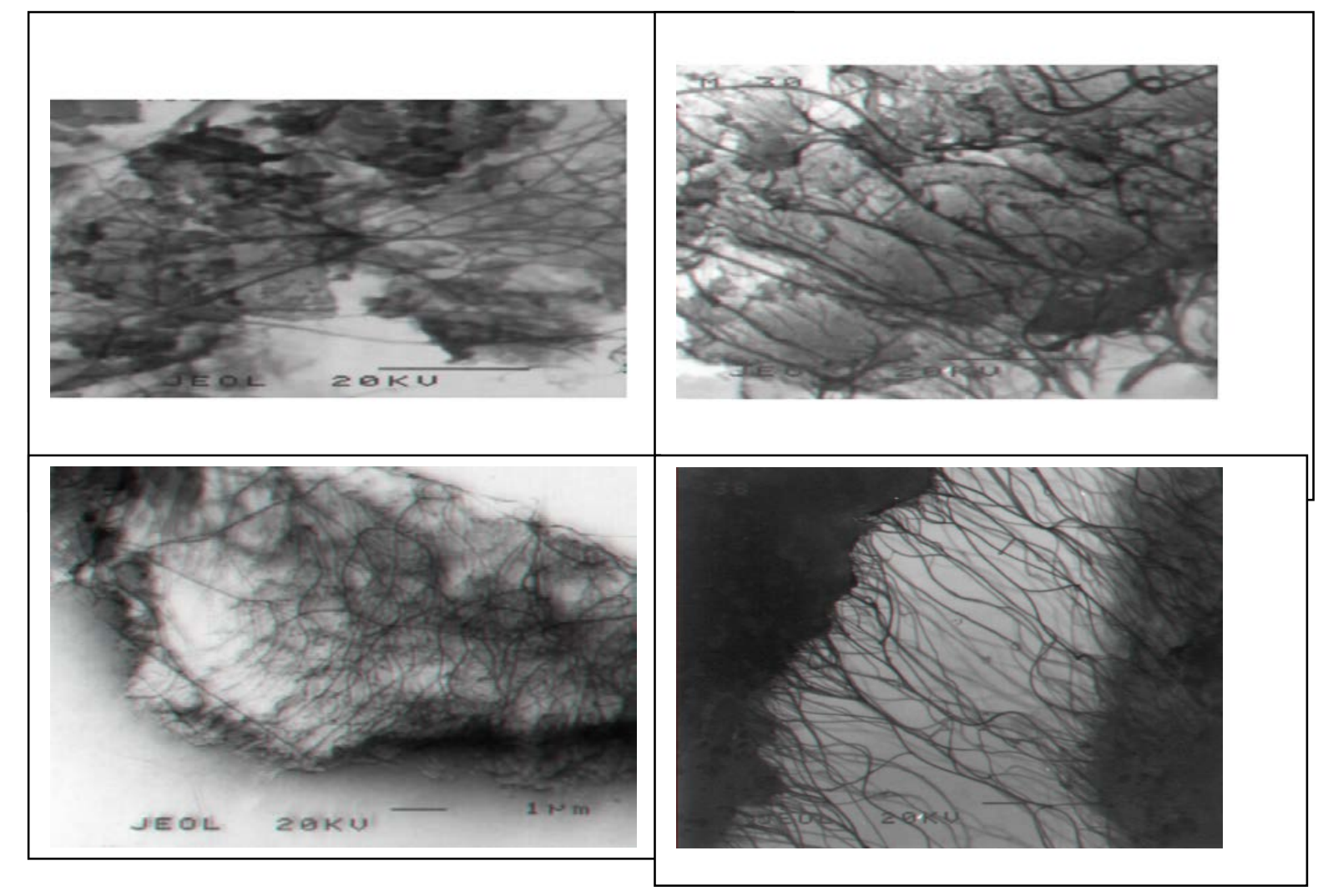

Fig 1. SEM images of nanotube sample (CVD). These images shows the presence of SWNT bundles everywhere in the sample on the surface and into the sample. Dark spots are due to carboneous materials.

TEM. The diameters of SWNTs within these bundles are estimated to be $1.05 \mathrm{~nm}$. In addition to the bundles of SWNTs, we observed individual SWNTs with average diameters of $1.5 \mathrm{~nm}$. The TEM image in Fig.4 also shows that the average size of the catalyst particles coated by amorphous carbon is about $7 \mathrm{~nm}$. 


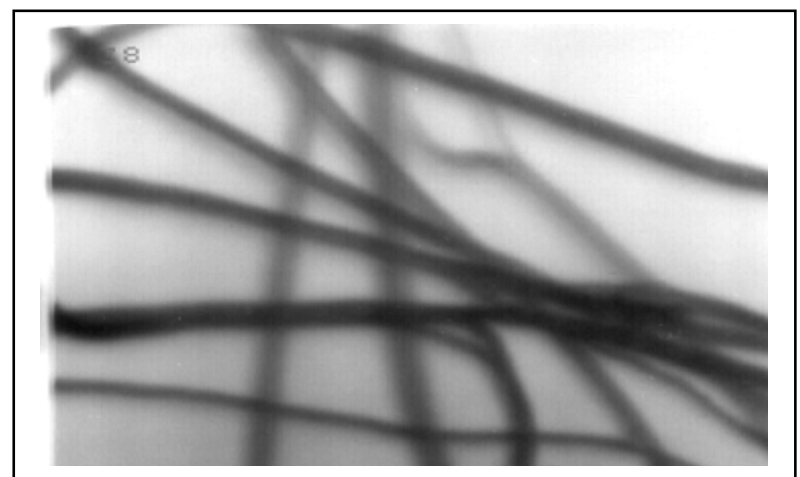

Fig 2. A SEM image of a sample, which shows clearly the size of the SWNT, bundles in the sample. As can be seen the diameter of the bundles
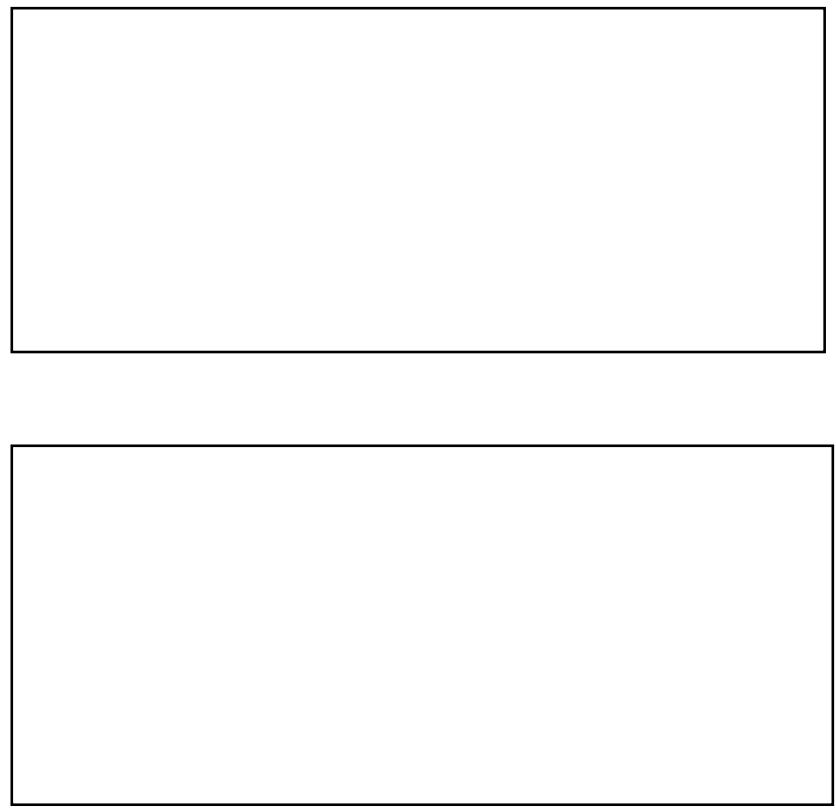

Fig 3. High-resolution TEM images of different areas of the sample, showing SWNT presence everywhere in the sample. The range of diameters of bundles are from 5.3 to $16 \mathrm{~nm}$. Dots spots are due to metal particles encapsulated in the nanotubes and amorphous carbon. 
Fig 4. High-resolution TEM image of the sample, clearly showing the size of the catalyst particles coated by amorphous carbon to

\subsection{Raman Spectra of SWNT samples}

Fig 5 shows the Raman spectra of the SWNT sample measured in the radial breathing

Raman intensity (a.u)
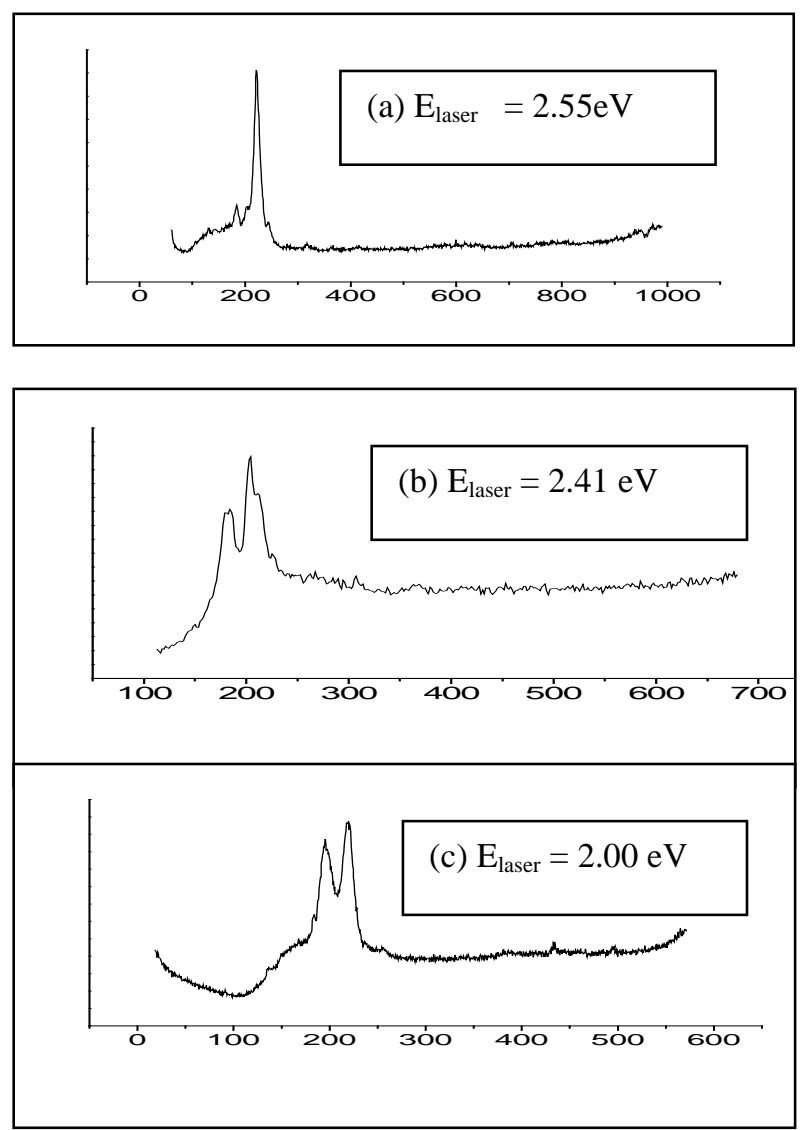

Fig 5. Raman spectra of the Radial Breathing Mode (RBM) of single wall carbon nanotubes with three laser lines. 
mode (RBM) range using three different laser lines. It is clear that the band shape is quite different from one line to another. This is because the sample contains carbon nanotubes with a large diameter distribution. The shapes of the RBM bands depend on the relative population of each nanotube type that is in resonance with the laser excitation frequency. In Fig.5a, which shows the response to the 2.54 ev incidence energy line, a large number of peaks, corresponding to a diameter distribution ranging from 1.1 to $1.31 \mathrm{~nm}$ are observed. From the diameter dependence of the allowed optical transitions [11], we can conclude that only semiconducting tubes with such diameters are being resonantly excited. This is in good agreement with the profile of the spectrum in the TM bands which shows a lorentzian lineshape. The spectrum measured at $2.41 \mathrm{ev}$ (Fig.5b) shows a similar profile. The peaks are shifted to lower frequencies and the range of diameters is from 1.13 to $1.35 \mathrm{~nm}$. The excited tubes are again semiconducting, in agreement with the TM mode results. The spectrum measured at 2 ev (Fig.5) shows tubes with sizes between 1.11 and $1.45 \mathrm{~nm}$. With such diameters, at this excitation energy, both metallic and semiconducting tubes are being resonantly excited. It is found that for higher wavelength laser lines, the peaks being excited shift to lower frequencies. Fig.6 shows the fitting of these three RBM spectra by lorentzian lines. The fitted peak frequencies are given in Table 1 together with their relative diameter sizes determined according to $(\mathrm{w}=232 / \mathrm{d}+6.5)$ and the possible chiral indices corresponding to these diameters are also given. 

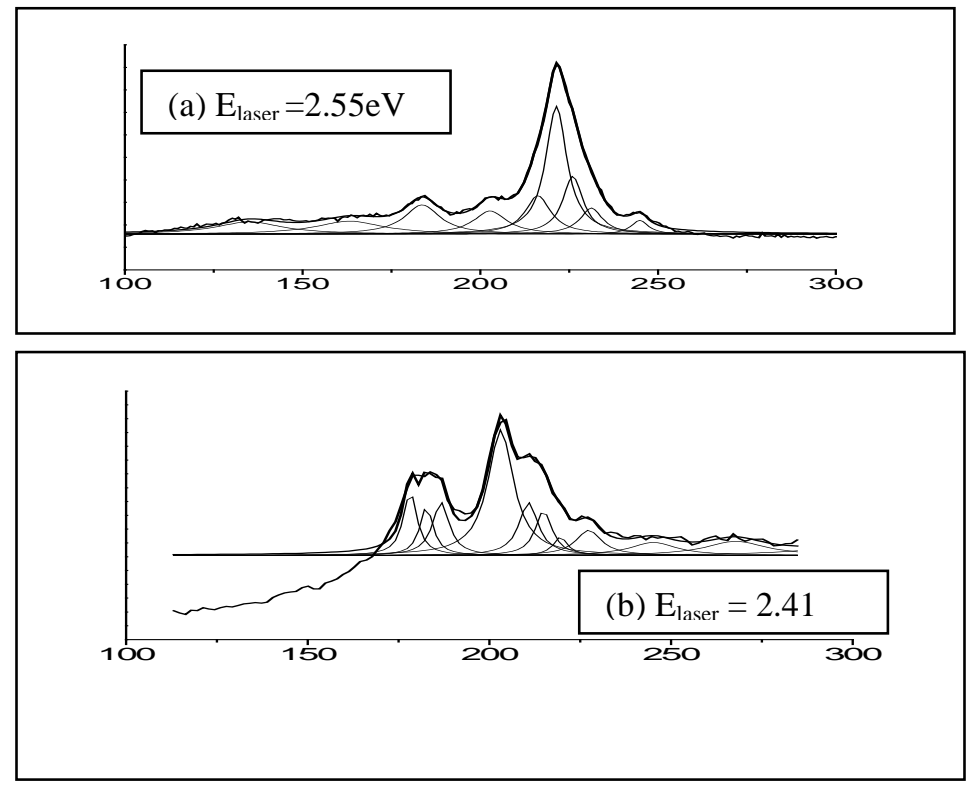

Table 1 : Peak frequencies (in $\mathrm{cm}^{-1}$ ) of the lorentzian curves used to fit the bands obtained with these laser excitation energies associated with the radial breathing mode (RBM) of single wall carbon nanotubes, together with possible chiral indices.

\begin{tabular}{cccccc}
\hline Laser excitation & \multicolumn{2}{c}{$2.55 \mathrm{ev}$} & $2.41 \mathrm{ev}$ & $2 \mathrm{ev}$ & possible \\
& $\mathrm{N}\left(\mathrm{cm}^{-1}\right) \mathrm{d}(\mathrm{nm})$ & $v\left(\mathrm{~cm}^{-1}\right)$ & $\mathrm{d}(\mathrm{nm})$ & $v\left(\mathrm{~cm}^{-1}\right) \mathrm{d}(\mathrm{nm})$ & $(\mathrm{n}, \mathrm{m})$ \\
\hline Line 1 & $\ldots$ & 268 & 0.89 & $\ldots$ & $(\underline{8,5})$
\end{tabular}




$\begin{array}{lccccccl}\text { Line 2 } & \ldots & & \ldots & & 254 & 0.94 & (10,3),(8,6) \\ \text { Line 3 } & 245 & 0.97 & 245 & 0.97 & \ldots & & (\underline{7,7}),(\underline{12,0}) \\ \text { Line 4 } & 231 & 1.03 & \ldots & & \ldots & & (13,0),(8,7) \\ \text { Line 5 } & 226 & 1.06 & 227 & 1.05 & \ldots & & (\underline{13,1}),(10,5) \\ \text { Line 6 } & 221 & 1.08 & \ldots & & 221 & 1.08 & (\underline{12,3}),(14,0) \\ \text { Line 7 } & \ldots & & 219 & 1.09 & \ldots & & (10,6),(9,7) \\ \text { Line 8 } & 216 & 1.11 & 215 & & 216 & 1.11 & (\underline{11,5)},(10,6),(13,2) \\ \text { Line 9 } & \ldots & & \ldots & & 211 & 1.13 & (14,1),(13,2) \\ \text { Line 10 } & 202 & 1.19 & 203 & 1.18 & 203 & 1.18 & (11,6),(10,7),(14,2) \\ \text { Line 11 } & \ldots & & \ldots & & 198 & 1.21 & (\underline{13,4}) \\ \text { Line 12 } & \ldots & & \ldots & & 193 & 1.24 & (\underline{9,9}) \\ \text { Line 13 } & \ldots & & 186 & 1.29 & \ldots & & (10,9),(14,4) \\ \text { Line 14 } & 183 & 1.31 & 182 & 1.32 & 183 & 1.31 & (12,7),(13,6) \\ \text { Line 15 } & \ldots & & 178 & 1.35 & \ldots & & (15,4),(11,9) \\ \text { Line 16 } & 163 & 1.48 & \ldots & & 166 & 1.45 & (13,8),(10,11),(14,7)\end{array}$

The G band Raman spectrum of graphite shows a single lorentzian peak at $1582 \mathrm{~cm}^{-1}$ with $\mathrm{E}_{2 \mathrm{~g}}$ symmetry [13] which results from the tangential stretching of the C-C bond atoms. In contrast to graphite, the G band of SWNT shows several peaks [14] with different symmetry: $A_{1 g}, E_{1 g}$ and $E_{2 g}$ [15]. In addition to this band there is the socalled D line which appears at a slightly lower frequency and is characteristic of graphite nanocrystals $[16,17]$. The G band of our SWNTs sample was probed using the same three laser energies and the results are shown in Fig.7. Fig.7a shows that the $\mathrm{G}$ band consists of two main components, one peak at $1594 \mathrm{~cm}^{-1}\left(\mathrm{G}^{+}\right)$and the other at $1560 \mathrm{~cm}^{-1}\left(\mathrm{G}^{-}\right)$. The $\mathrm{G}^{+}$is associated with carbon atom vibrations along the nanotube axis. The $\mathrm{G}^{-}$band is due to vibrations of carbon atoms along the circumferential direction of the SWNT [18], and shows whether the SWNT is metallic (Breit-WignerFano lineshape) or semi-conducting (lorentzian lineshape) [12,19]. This spectrum also shows a third peak at $1349 \mathrm{~cm}^{-1}$ which indicates the presence of carbon nanoparticles and amorphous carbon in the sample. It is found that, on going from an incident beam energy of $2.55 \mathrm{ev}$ to one of $2 \mathrm{ev}$, the weak peak changes to a shoulder on the left of the profile; also a slight asymmetry is observed, which is assigned to the contribution 
of metallic tubes. Moreover, using this excitation wavelength $(\lambda=632.8 \mathrm{~nm})$, the peak due to carbon nanoparticles (D-line) is found at $1328 \mathrm{~cm}^{-1}$. Figure 8 shows the fitting of these three TM spectra from SWNT by a lorentzian lines. The frequencies of the peaks used for the fits are shown in Table 2.

Table 2 : Frequencies (in $\mathrm{cm}^{-1}$ ) of the lorentzian curves used to fit the bands associated with the tangential mode (TM) of single wall carbon nanotubes, obtained with the three laser excitation energies quoted.

\begin{tabular}{|c|c|c|c|}
\hline Laser excitation & $2.55 \mathrm{ev}$ & $2.41 \mathrm{ev}$ & $2 \mathrm{ev}$ \\
\hline Line 1 & 1614 & 1616 & $\ldots$. \\
\hline Line 2 & $\ldots$ & $\ldots$ & 1612 \\
\hline Line 3 & 1601 & $\ldots$ & $\ldots$ \\
\hline Line 4 & $\ldots$ & 1598 & $\ldots$ \\
\hline Line 5 & $\ldots$ & $\ldots$ & 1594 \\
\hline Line 6 & 1590 & $\ldots$ & $\ldots$ \\
\hline Line 7 & $\ldots$ & $\ldots$ & 1584 \\
\hline Line 8 & $\ldots$ & 1569 & \\
\hline Line 9 & 1564 & $\ldots$ & 1566 \\
\hline Line 10 & 1542 & $\ldots$. & $\ldots$ \\
\hline Line 11 & $\ldots$ & 1538 & 1537 \\
\hline
\end{tabular}

From Table 2 we observed that the G band of SWNT in the bundle is composed of four $\left(E_{\text {laser }}=2.41 \mathrm{ev}\right)$ and five (both $E_{\text {laser }}=2.55$ and $2 \mathrm{ev}$ ) lorentsian components. The symmetry assignments to the lorentsian components for each laser energy are:

$$
\begin{aligned}
& \mathbf{E}_{\text {laser }}=2.55 \mathrm{ev}: \omega_{E 2}^{+}=1614 \mathrm{~cm}^{-1}\left(\mathrm{E}_{2 \mathrm{~g}}\right), \omega_{E 2}^{+}=1601 \mathrm{~cm}^{-1}\left(\mathrm{E}_{2 \mathrm{~g}}\right), \omega_{G}^{+}=1590 \mathrm{~cm}^{-1}\left(\mathrm{~A}_{1 \mathrm{~g}}, \mathrm{E}_{1 \mathrm{~g}}\right) \\
& \omega_{G}^{-}=1564 \mathrm{~cm}^{-1}\left(\mathrm{~A}_{1 \mathrm{~g}}, \mathrm{E}_{1 \mathrm{~g}}\right) \text { and } \omega_{E 2}^{-}=1542 \mathrm{~cm}^{-1}\left(\mathrm{E}_{2 \mathrm{~g}}\right) . \\
& \mathrm{E}_{\text {laser }}=2.41 \mathrm{ev}: \omega_{E 2}^{+}=1616 \mathrm{~cm}^{-1} \omega_{G}^{+}=1598 \mathrm{~cm}^{-1}, \omega_{G}^{-}=1569 \mathrm{~cm}^{-1} \text { and } \omega_{E 2}^{-}=1538 \mathrm{~cm}^{-1} . \\
& \mathbf{E}_{\text {laser }}=2 \mathbf{e v}: \omega_{E 2}^{+}=1612 \mathrm{~cm}^{-1}, \omega_{G}^{+}=1594 \mathrm{~cm}^{-1}, \omega_{G}^{+}=1584 \mathrm{~cm}^{-1} \text { for metallic nanotubes }
\end{aligned}
$$
present in SWNT bundles [20], $\omega_{G}^{-}=1566 \mathrm{~cm}^{-1}$ and $\omega_{E 2}^{-}=1537 \mathrm{~cm}^{-1}$.

For comparison, we have also measured the Raman spectra of Hipco* SWNT material purchased from Carbon Nanotechnology Incorporated ${ }^{2}$. The Raman spectrum measurements reported here (RBM and TM modes) were performed on this material 
purified by the remove of large catalyst particles. Fig 9 shows the Raman spectrum of Hipco SWNT measured in the radial breathing mode range using two different laser energies (2.41, 2 ev). Fig 9a shows RBM measurements with the higher energy. A large number of peaks are observed which correspond to a diameter distribution ranging from 0.9 to $1.47 \mathrm{~nm}$. Figure $9 \mathrm{~b}$ shows the spectrum measured with the lower incident energy. Here, the peaks are shifted to higher frequencies and the range of diameters goes from 0.82 to $1.32 \mathrm{~nm}$. This is in contrast to our SWNT sample produced by the CVD method, because in that case when the laser energy gets smaller, the observed peaks are shifted to lower frequencies. Perhaps this different behaviour is related to the different electronic nature of the nanotubes in two samples. In the Hipco sample, the majority of nanotubes excited by these two laser energies

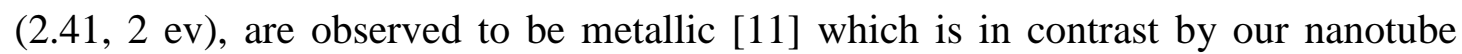
(CVD) sample where the majority of excited nanotubes are semiconducting. The fitted Peak frequencies together with their relative diameters and corresponding chiral indices are given in Table 3. The results suggest that both samples have roughly the same distribution of diameters but with the Hipco material showing a slightly wider range of diameters

\footnotetext{
* This material is produced by a high pressure carbon monoxide technique.

Table 3: Frequencies (in $\mathrm{cm}^{-1}$ ) of RBM bands of Hipco SWNT together with corresponding diameters and chiral indices.
}

\begin{tabular}{lcccll}
\hline Laser excitation & \multicolumn{2}{c}{$2.41 \mathrm{ev}$} & \multicolumn{2}{c}{$2 \mathrm{ev}$} & possible $(\mathrm{n}, \mathrm{m})$ \\
& $v\left(\mathrm{~cm}^{-1}\right)$ & $\mathrm{d}(\mathrm{nm})$ & $v\left(\mathrm{~cm}^{-1}\right)$ & $\mathrm{d}(\mathrm{nm})$ & \\
\hline$\ldots$ & $\ldots$ & 294 & 0.82 & $(7,5),(8,4)$ \\
$\ldots$ & $\ldots$ & 281 & 0.86 & $(\underline{9,3})$ \\
270 & 0.9 & $\ldots$ & $\ldots$ & $(\underline{8,5})$ \\
$\ldots$ & $\ldots$ & 255 & 0.95 & $(\underline{7,7}),(12,0)$ \\
235 & 1.04 & $\ldots$ & $\ldots$ & $(\underline{9,6})$ \\
$\ldots$ & $\ldots$ & 227 & 1.07 & $(\underline{13,1}),(\underline{8,8})$ \\
$\ldots$ & $\ldots$ & 217 & 1.13 & $(\underline{11,5})$
\end{tabular}




$\begin{array}{ccccl}214 & 1.14 & \ldots & \ldots & (14,1) \\ 195 & 1.26 & \ldots & \ldots & (11,7),(14,3),(15,2) \\ \ldots & \ldots & 193 & 1.27 & (\underline{12,6)}),(11,8),(14,5) \\ \ldots & \ldots & 186 & 1.32 & (\underline{15,3}),(112,7) \\ 184 & 1.34 & \ldots & \ldots & (13,6),(12,5) \\ 176 & 1.4 & \ldots & \ldots & (14,6),(15,5) \\ 169 & 1.47 & \ldots & \ldots & (14,7),(13,8)\end{array}$

The $\mathrm{G}$ band of the Hipco sample was also probed using two different laser energies (2.41, $2 \mathrm{ev).} \mathrm{The} \mathrm{results} \mathrm{are} \mathrm{shown} \mathrm{in} \mathrm{Fig} \mathrm{10.} \mathrm{It} \mathrm{is} \mathrm{clear} \mathrm{that,} \mathrm{on} \mathrm{going} \mathrm{from} \mathrm{incident}$ beam energy $2 \mathrm{ev}$ to $2.41 \mathrm{ev}$, the weak peak changes to a shoulder on the left of the profile. Fits to these TM spectra using lorentzian line shapes are shown in Fig 11 and the corresponding frequencies are given in the TABLE 4.

Table 4: Frequencies (in $\mathrm{cm}^{-1}$ ) of the lorentzian curves used to fit the bands associated with the tangential mode (TM) of Hipco nanotube, obtained with two laser excitation line.

\begin{tabular}{lcc}
\hline Laser excitation & $2.41 \mathrm{ev}$ & $2 \mathrm{ev}$ \\
& & \\
\hline Line 1 & $\ldots$. & 1591 \\
Line 2 & 1583 & $\ldots$ \\
Line 3 & 1558 & $\ldots$. \\
Line 4 & $\ldots$. & 1553 \\
Line 5 & $\ldots$. & 1540 \\
Line 6 & 1534 & $\ldots$. \\
Line 7 & $\ldots$. & $\ldots \ldots$
\end{tabular}

We can also compare the structure of our SWNT sample produced by the CVD method with two samples reported in the literature which were produced by other methods namely Laser Vaporisation (LA) and Electric Arc Discharge (EA). In the RBM and TM modes we found that the frequencies observed for our SWNT sample appear at higher frequencies than those observed in the LA [21,22] and EA [22-24] materials. We can therefore conclude that the diameters of our SWNTs are smaller. The diameters are also somewhat smaller than for the Hipco material. As the 
production of SWNTs with small diameters could be important for different possible applications, this might be a useful advantage of the CVD method.

\section{Conclusions}

A large number of SWNT bundles were observed in both samples using SEM and TEM imaging.. SEM images show the presence of SWNT bundles with a range of diameters throughout the samples. The diameters of the bundles lie between 10 and 70 nm. The images also show many dark dots and shaded area which are respectively metallic particles encapsulated within the nanotubes and amorphous carbon deposits. The TEM images show that the bundles have diameters between 5.3 and $16 \mathrm{~nm}$ and lengths up to tens of micrometers. The average diameter of SWNTs within these bundles is estimated to be about $1.05 \mathrm{~nm}$. The difference between bundle size in the SEM and TEM images may be related to the sonication process used for samples prepared for the TEM. Besides the bundles of SWNTs, the individual SWNTs with average diameters of $1.5 \mathrm{~nm}$ are observed. The TEM image also shows that the average size of the carboneous deposits is about $7 \mathrm{~nm}$.

Because the samples contain carbon nanotubes with large diameter distributions, the shape of the RBM bands are quiet different from one line to another. Therefore we used three different incident energies (2.54, 2.41 and 2ev) to cover all nanotubes diameters. Measurement with $2.54 \mathrm{ev}$ incident energy shows that the range of diameter distribution is between 1.1 to $1.31 \mathrm{~nm}$. Therefore, only semiconducting tubes of such diameters can be resonantly excited. This is in agreement with the profile of the spectrum in the TM mode, which shows a lorentzian lineshape. The spectrum measured at $2.41 \mathrm{ev}$ shows the peaks are shifted to lower frequencies and the range of diameters here is from 1.13 to $1.35 \mathrm{~nm}$. The excited tubes are semiconducting. 
Measurement with 2ev incident energy shows that the size of tubes is between 1.11 to $1.45 \mathrm{~nm}$ and here both metallic and semiconducting tubes are observed. It is found that for lower incident energies, the peaks shift to lower frequencies. From our observation of the Raman spectra, we can conclude for several reasons that the concentration of armchair tubes in sample must be very low. Raman spectra of Hipco SWNT were measured in both the RBM and TM modes using two different incident energies (2.41 and 2ev). Measurement with $2.41 \mathrm{ev}$ incident energy shows that the diameter distribution is from 0.9 to $1.47 \mathrm{~nm}$. For $2 \mathrm{ev}$ incident energy, the peaks are shifted to higher frequencies and the range of diameters is from 0.82 to $1.32 \mathrm{~nm}$. It is clear that the distribution of diameters is wider for the Hipco nanotube sample than for our nanotube (CVD) sample at the two incident energies (2.41, $2 \mathrm{ev).} \mathrm{Also} \mathrm{it} \mathrm{is} \mathrm{shown} \mathrm{that}$ ,on going from energy $2.41 \mathrm{ev}$ to $2 \mathrm{ev}$, the bands for our nanotube (CVD) sample are shifted to lower frequencies with respect to Hipco nanotube sample. The different behaviour between Hipco and our sample may be related to the electronic nature of nanotubes in two samples. From comparison of our sample with samples produced with EA and LA methods we can conclude that the range of our SWNTs diameter produced with CVD method are smaller than the EA and LA methods.

\section{Acknowledgement}

The authors would like to thank Dr. C. A. Faunce for his assistance with the SEM and TEM observations.

${ }^{1}$ Fisher Chemical (www.fisher.co.uk

${ }^{2}$ Carbon Nanotechnology Incorporated (www.cnanotech.com)

\section{References}


[1] Iijima S., Helical microtubules of graphitic carbon,. Nature 354 (1991), p. 56.

[2] Dresselhaus MS, Dresselhaus G, Eklund PC., Science of Fullerenes and Carbon Nanotubes, Academic Press: San Diego (1996).

[3] Guo T, Nikolaev P, Thess A, Colbert DT, Smalley RE., Catalytic growth of Single- walled nanotubes by laser Vaporisation, Chem Phys Lett 243 (1995), pp. 4954.

[4] Journet C, Bernier P., Production of Carbon Nanotubes, Appl Phys A 67 (1998), pp.1-9.

[5] Dai H, Rinzler AG, Nikolaev P, Thess A, Colbert DT, Smalley RE., Siingle-wall nanotubes produced by metal-catalyzed disproportionation of carbon monoxide, Chem Phys Lett 260 (1996), pp. 471-475.

[6] Richter E, Subbaswany KR., Theory of Size-Dependent Resonance Raman Scattering from Carbon Nanotubes, Phys Rev Lett 79 (1997) pp. 2738-2740

[7] Alvarez L, Righi A, Rols S, Anglaret E, Sauvajol JL.,.Excitation energy dependence of the Raman spectrum of single-walled carbon nanotubes, Chem Phys Lett 320 (2000) pp. 441-447.

[8] Cheng HM, Li F, Su G, Pan HY, He LL, Sun X, et al., Large-scale and low-cost synthesis of single-walled carbon nanotubes by the catalytic pyrolysis of hydrocarbons, Appl Phys Lett 72 (1998), pp.3282-3284.

[9] Colomer JF, Benoit JM, Stephan C, Lefrant S, Van Tendeloo G, Nagy JB., Characterization of single-wall carbon nanotubes produced by CCVD method, Chem Phys Lett 345 (2001), pp. 11-17.

[10] Anglaret E, Bendiab N, Guillard T, Journet C, Flamant G, Laplaze D, et al., Raman characterization of single wall carbon nanotubes prepared by the solar energy route, Carbon 36 (1998) (12), pp1815-1820. 
[11] Alvarez L, Righi A, Guillard T, Rols S, Anglaret E, Laplaze D et al., Resonant Raman study of the structure and electronic properties of single-wall carbon nanotubes, Chem Phys Lett 316 (2000), pp. 186-190.

[12] Brown SDM, Jorio A, Corio P, Dresselhaus MS, Dresselhaus G, Saito R et al., Origin of the Breit-Wigner-Fano lineshape of the tangential G-band feature of metallic carbon nanotubes, Phys Rev B 63 (2001) 155414.

[13] Jorio A, Saito R, Dresselhaus G, Dresselhaus MS, Determination of nanotubes properties by Raman spectroscopy, Phil. Trans. R. Soc. Lond. A 362 (2004), pp. 23112336

[14] Duesberg GS, Loa I, Burghard M, Syassen K, Roth S., Polarized Raman Spectroscopy on Isolated Single-Walled Carbon Nanotubes, Phy Rev Lett 85 (2000) pp.5436-5438.

[15] Jorio A, Dresselhaus G, Dresselhaus MS, Souza M, Dantas MSS, Pimenta MA, et al., Polarized Raman Study of Single-Wall Semiconducting Carbon Nanotubes, Phy Rev Lett 85 (2000), pp. 2617-2619.

[16] Hiura H, Ebbesen TW, Takahashi KT. Raman studies of Carbon Nanotubes. Chem Phys Lett 202 (1993), pp.509-511.

[17] Pimenta MA, Marucci A, Empedocles SA, Bawendi MG, Hanlon EB, Rao AM, et al., Raman modes of metallic carbon, Phys Rev B 54(24) (1998),pp.R16016R16019.

[18] Saito R, Dresselhaus G, Dresselhaus MS., In: Physical Properties of Carbon Nanotubes; Imperial College Press (London) 1998

[19] Gregg SJ, Sing KSW. In: Adsorption, Surface area and Porosity; 1982 
[20] Jorio A, Filho GS, Dresselhaus G, Dresselhaus MS, Swan AK, et al., G-band resonant Raman study of 62 isolated single-wall carbon nanotubes, Phy Rev B 65 (2002), pp.155412-

[21] Pimenta MA, Marucci A, Brown SDM, Matthews MJ, Rao AM, Eklund PC and et al., Resonant Raman effect in single-wall carbon nanotubes, J. Mater. Res 13 (1998) pp.2396

[22] Sauvajol JL, Anglaret E, Rols S, Journet C, Goze C, Bernier P et al., Structure and vibrational properties of single wall carbon nanotubes, Synthetic Metals $\mathbf{1 0 3}$ (1999), pp.2537-2539.

[23] Chapelle ML, Lefrant S, Journet C, Maser W, Bernier P, Loiseau A., Raman studies on single walled carbon nanotubes produced by the Electric Arc Technique, Carbon 36 (1998), pp.705-708.

[24] Journet C, Alvarez L, Micholet V, Guillard T, Chapelle ML, Anglaret E et al., Single wall carbon nanotubes : Two ways of production, Synthetic Metals 103 (1999) pp.2488-2489.

\section{Table Headings}

Table 1 : Peak frequencies (in $\mathrm{cm}^{-1}$ ) of the lorentzian curves used to fit the bands obtained with these laser excitation energies associated with the radial breathing mode (RBM) of single wall carbon nanotubes, together with possible chiral indices.

Table 2 : Frequencies (in $\mathrm{cm}^{-1}$ ) of the lorentzian curves used to fit the bands associated with the tangential mode (TM) of single wall carbon nanotubes, obtained with the three laser excitation energies quoted.

Table 3: Frequencies (in $\mathrm{cm}^{-1}$ ) of RBM bands of Hipco SWNT together with corresponding diameters and chiral indices.

Table 4: Frequencies (in $\mathrm{cm}^{-1}$ ) of the lorentzian curves used to fit the bands associated with the tangential mode (TM) of Hipco nanotube, obtained with two laser excitation line. 


\section{Figure Captions}

Fig 1. SEM images of nanotube sample (CVD). These images shows the presence of SWNT bundles everywhere in the sample on the surface and into the sample. Dark spots are due to carboneous materials.

Fig 2. A SEM image of a sample, which shows clearly the size of the SWNT, bundles in the sample. As can be seen the diameter of the bundles lie between 10 and $70 \mathrm{~nm}$. Fig 3. High-resolution TEM images of different areas of the sample, showing SWNT presence everywhere in the sample. The range of diameters of bundles are from 5.3 to $16 \mathrm{~nm}$. Dots spots are due to metal particles encapsulated in the nanotubes and amorphous carbon.

Fig 4. High-resolution TEM image of the sample, clearly showing the size of the catalyst particles coated by amorphous carbon to be about $7 \mathrm{~nm}$.

Fig 5. Raman spectra of the Radial Breathing Mode (RBM) of single wall carbon nanotubes with three laser lines.

Fig 6. Fitting of the RBM mode of SWNT with laser lines 2.55, 2.41 and 2 ev by a sum of lorentzian lines.

Fig 7. Raman spectra of the tangential mode (TM) of SWNT sample with three laser line.

Fig 8. Fitting of the (TM) mode of SWNT with three laser lines 2.55, 2.41 and 2ev by a sum of lorentzian lines.

Fig 9. Raman spectra of the Radial Breathing mode (RBM) of Hipco nanotube With two laser lines (a) 2.41ev and (b) 2ev 
Fig 10. Raman spectra of the tangential mode (TM) of Hipco nanotube with two laser line (a) 2.41ev and (b) 2ev 